\title{
Sources of negative tunneling magnetoresistance in multilevel quantum dots with ferromagnetic contacts
}

\author{
Sonja Koller and Milena Grifoni \\ Theoretische Physik, Universität Regensburg, DE-93040 Regensburg, Germany
}

Jens Paaske

The Niels Bohr Institute and Nano-Science Center, University of Copenhagen, Universitetsparken 5, DK-2100 Copenhagen Ø, Denmark

(Received 12 August 2011; revised manuscript received 23 November 2011; published 13 January 2012)

\begin{abstract}
We analyze distinct sources of spin-dependent energy level shifts and their impact on the tunneling magnetoresistance (TMR) of interacting quantum dots coupled to collinearly polarized ferromagnetic leads. Level shifts due to virtual charge fluctuations can be quantitatively evaluated within a diagrammatic representation of our transport theory. The theory is valid for multilevel quantum dot systems and we exemplarily apply it to carbon nanotube quantum dots, where we show that the presence of many levels, among them of excited states, can qualitatively influence the TMR effect.
\end{abstract}

DOI: 10.1103/PhysRevB.85.045313

PACS number(s): 73.63.Kv, 73.23.Hk

\section{INTRODUCTION}

Recent transport experiments on quantum dots coupled to ferromagnetic leads have demonstrated the existence of spin-dependent energy level shifts through the observation of negative tunnel magnetoresistance (TMR) effects in the single electron tunneling regime $^{1}$ and spin splitting in the Kondo regime. ${ }^{2-5}$ So far qualitatively different approaches for explaining the origin of the underlying shifts coexist. For example, negative TMR data from carbon nanotube (CNT) measurements have been fitted with a model relying on spin-dependent interfacial phase shifts $^{6}$ picked up by the wave function during multiple reflections at a spin-active interface. ${ }^{7}$ The concept is related to that of spin-mixing conductance $^{8}$ and, because it only depends on the properties of the spin-active barrier region, is only weakly gate dependent and present in both interacting and noninteracting systems. ${ }^{9}$ In contrast, experiments on $\mathrm{CNTs}^{3,5}$ and InAs nanowires ${ }^{4}$ in the Kondo regime have demonstrated a combination of gate-dependent and gate-independent contributions to the energy level shifts. Both can be explained in terms of charge fluctuations, ${ }^{10}$ whereby electron-electron interactions are responsible for the logarithmic gate dependence, ${ }^{11}$ while a Stoner splitting of the energy bands of the magnetically polarized leads accounts for the almost gate-independent part. $^{12,13}$

While the effects of the energy level shifts in the Kondo regime are by now well understood, a thorough understanding of their influence on the TMR phenomenon in interacting quantum dots is still missing. The negative TMR data ${ }^{1}$ have been satisfactorily fitted in terms of a generalized Anderson model already including gate-independent level shifts. In Ref. 14 a reflection Hamiltonian was included to account for reflection processes at the interface in a second-order sequential tunneling theory.

In this work, we specifically address the TMR phenomenon within a diagrammatic approach to the reduced density matrix of the nanosystem ${ }^{15}$ and discuss different level-shift-induced mechanisms for negative TMR. Spin-dependent level shifts can originate to the lowest order in the tunneling Hamiltonian (sequential tunneling limit) if off-diagonal elements of the reduced density matrix participate in the dynamics. This is the case for noncollinearly polarized leads ${ }^{14,16,17}$ but also for collinearly polarized leads for some double-dot setups. ${ }^{18}$ Here we consider situations where coherences of the reduced density matrix do not play a crucial role in the dynamics. We rather identify charge fluctuation terms in all orders in the tunneling Hamiltonian which sum up to a Taylor series yielding intrinsic level shifts.

In particular we discuss two types of spin-dependent level shifts: strongly gate-dependent ones deriving from a net difference of the majority and minority density of states at the Fermi level, and largely gate-independent ones caused by a Stoner shift of the majority and minority bands in the leads. Our results are valid for generic multilevel quantum dots and reproduce the results of Ref. 12 in the case of a simple Anderson model.

Finally, we analyze the TMR of a CNT quantum dot and show that, due to the multilevel spectrum, the intrinsic contributions give rise to a marked gate voltage dependence and a TMR which can indeed become negative. Moreover, we show that charge fluctuations to states including bosonic excitations, which are present in CNTs due to the linearity of the noninteracting spectrum, can largely influence the gate voltage dependence of the level shifts.

The paper is structured as follows: Sec. II contains a discussion of possible mechanisms for negative TMR. In Sec. III the model Hamiltonian is elucidated, while in Sec. IV the level shifts are calculated analytically. Results for the conductance and TMR of armchair CNTs are presented in Sec. V, while in Sec. VI conclusions are drawn.

\section{MECHANISMS OF NEGATIVE TMR}

The basic mechanism underlying a negative TMR is the presence of an effective generalized Zeeman field $h^{P / A}\left(V_{g}\right)$ accounting for both extrinsic (stray fields, applied magnetic field) and intrinsic sources of spin-dependent level shifts. Here $(P / A)$ refers to contacts with parallel/antiparallel 


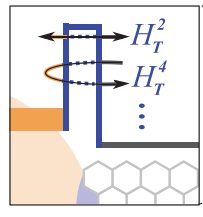

band schematics
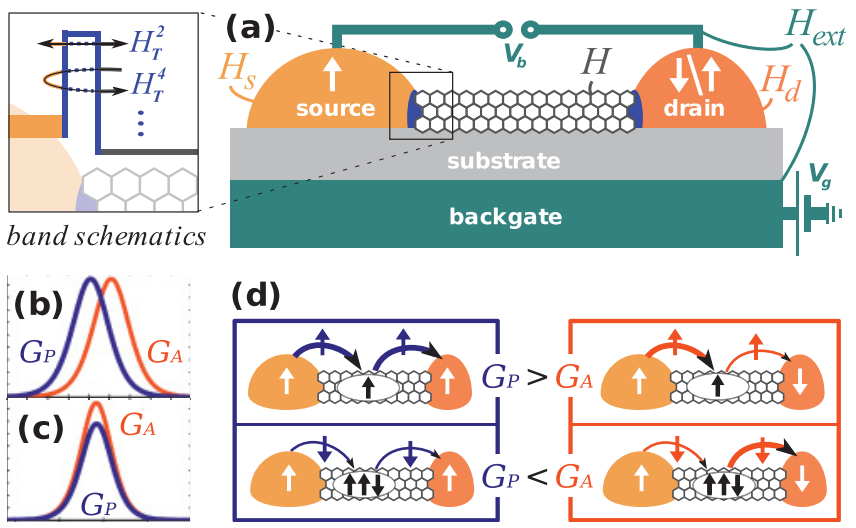

(d)

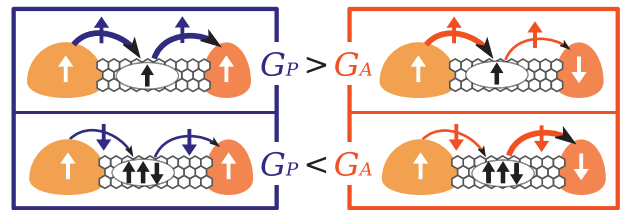

FIG. 1. (Color online) (a) Transport setup of a CNT quantum dot with ferromagnetic leads. If an electron enters the barrier region at the tube ends, it can either tunnel to the lead or be reflected at the interface. Tunneling processes in the contact region are described by even powers of the tunneling Hamiltonian $H_{T}$. (b) and (c) Conductances $G_{P}$ and $G_{A}$ versus gate voltage for two situations exhibiting negative TMR. (d) The negative TMR mechanism related to panel (c), where thick/thin lines describe processes that are favored/disfavored being associated with majority/minority spins.

magnetization (see Fig. 1). For spin-dependent transport experiments, typically two ferromagnetic contacts of different widths [compare Fig. 1(a)] are utilized to obtain a hysteretic polarization behavior when exposing the setup to a sweep of an external magnetic field. Due to remanent magnetization, the polarization of the contacts is inverted only at a reverted field of some hundreds of millitesla in strength, until the magnetically softer of the two contacts switches first to align with the external field. Thus, for some range of the field, the contacts stay in antiparallel configuration. Intuitively, one would expect that the conductance for contacts polarized in parallel $\left(G_{P}\right)$ is larger than the one for the antiparallel case $\left(G_{A}\right)$, yielding positive values of TMR $:=\left(G_{P} / G_{A}\right)-1$. Nevertheless, there are at least two different mechanisms which can lead to a negative TMR, as sketched in Figs. 1(b) and $1(\mathrm{c})$, respectively. Here we consider quantum dots in the single electron tunneling regime and look at the linear conductance as a function of gate voltage $V_{g}$. For sufficiently small couplings the temperature determines the width of the conductance peaks, while the peak position signals at which value of $V_{g}$ the two many-body ground state configurations with $N$ and $N+1$ electrons involved in the transition have the same electrochemical potential: $\mu\left(N, V_{g}\right)=\mu\left(N+1, V_{g}\right)$. Because the effective Zeeman field leads to corrections to the energy difference $E_{b}-E_{a}:=E_{b a}$ associated with the transition from a many-body state $a$ to a many-body state $b$, this in turn modifies the position of the conductance peaks.

First, and most obviously, there can be negative TMR if there is a noticeable offset in the conductance peak positions in parallel and antiparallel configuration [see Fig. 1(b)]. This requires $\left|h^{P}-h^{A}\right|$ of the order of the width $k_{B} T$ of the conductance peaks. Second, however, negative TMR can also arise for $\left|h^{P}\right|=\left|h^{A}\right| \approx k_{B} T$ [see Fig. 1(c)]. This is because the effective magnetic field responsible for the effective Zeeman
TABLE I. (Color online) Ground states of a CNT in shell $n$ accounting for spin $\sigma=\{\uparrow, \downarrow\}$ and orbital degrees of freedom $\tilde{r}=$ $\{+/-\}$, leading to four-electron shells. The number of electrons in band $\tilde{r}$ with spin $\sigma$ is $N_{\tilde{r} \sigma}$ and in the $\operatorname{dot} N=\sum_{\tilde{r} \sigma} N_{\tilde{r} \sigma}$. With $h^{P / A} \neq 0$, only the highlighted (gray) states are ground states.

\begin{tabular}{cc}
\hline \hline \multicolumn{1}{c}{ Filling } & Ground state(s) \\
\hline \hline$N=4 n$ & $|\cdot, \cdot\rangle$ \\
$N=4 n+1$ & $|\uparrow, \cdot\rangle,|\cdot, \uparrow\rangle,|\downarrow, \cdot\rangle,|\cdot, \downarrow\rangle$ \\
$N=4 n+2$ & $|\uparrow, \uparrow\rangle,|\uparrow \downarrow, \cdot\rangle,|\uparrow, \downarrow\rangle,|\cdot, \uparrow \downarrow\rangle,|\downarrow, \uparrow\rangle,|\downarrow, \downarrow\rangle$ \\
$N=4 n+3$ & $|\uparrow \downarrow, \uparrow\rangle,|\uparrow, \uparrow \downarrow\rangle,|\uparrow \downarrow, \downarrow\rangle,|\downarrow, \uparrow \downarrow\rangle$ \\
\hline \hline
\end{tabular}

shift also removes the spin-degeneracy of the ground states by favoring the states with maximum total spin. This situation is illustrated in Fig. 1(d) for the case of a CNT quantum dot, for which we consider the CNT Hamiltonian, Eq. (2). The four-electron shells of the dot are filled sequentially upon sweeping the gate voltage and only a single shell needs to be considered at a time. The case of orbital $(\Delta \varepsilon=0)$ and spin degeneracy is illustrated for the $n$th shell in Table I. For the $4 n \leftrightarrow 4 n+1$ and $4 n+1 \leftrightarrow 4 n+2$ transitions and $h^{P / A} \neq 0$ only spin-up electrons are required. Hence, due to the larger density of spin-up electrons, peak heights for parallel contact polarization will exceed those for the antiparallel case. On the other hand, for the $4 n+2 \leftrightarrow 4 n+3$ and $4 n+3 \leftrightarrow$ $4 n+4$ transitions spin-down electrons have to be transferred through the dot. In this latter case, a configuration with antiparallel contact polarization might be favored, leading to $G_{A}>G_{P}$.

\section{THE MODEL HAMILTONIAN}

For a quantitative description we consider the transport setup of Fig. 1. In the limit of weak coupling to the leads it can be described by the total Hamiltonian

$$
\hat{H}_{\mathrm{tot}}^{P / A}=\hat{H}+\hat{H}_{\mathrm{ext}}^{P / A}+\sum_{l=s, d} \hat{H}_{l}+\hat{H}_{T},
$$

where $\hat{H}=\hat{H}_{0}-e \alpha V_{g} \hat{N}$ comprises the Hamiltonian $\hat{H}_{0}$ of the isolated quantum dot and the effects of a gate voltage ( $\alpha$ is a conversion factor of the order of one). In the case of an armchair nanotube quantum dot of medium-to-large radius far from half-filling it reads ${ }^{19}$

$$
\hat{H}_{0}=\frac{1}{2} E_{c} \hat{N}^{2}+\frac{1}{2} \sum_{\tilde{r}, \sigma}\left(\varepsilon_{0} \hat{N}_{\tilde{r} \sigma}+\tilde{r} \Delta \varepsilon\right) \hat{N}_{\tilde{r} \sigma}+\hat{H}_{B},
$$

where $\varepsilon_{0}=\hbar v_{\mathrm{F}} / \pi L$, with $v_{F}$ being the Fermi velocity and $L$ the CNT length, is the level spacing; $\Delta \varepsilon$ is the orbital mismatch; and $E_{c}$ is the charging energy. The number of electrons in the orbital band $\tilde{r}= \pm$ with spin $\sigma$ is determined by the number operator $\hat{N}_{\tilde{r} \sigma}$ and the total number is determined by $\hat{N}=\sum_{\tilde{r} \sigma} \hat{N}_{\tilde{r} \sigma}$. Finally,

$$
\hat{H}_{B}=\sum_{n \neq 0} \sum_{j=c, s} \varepsilon_{j, n} \hat{a}_{j n}^{\dagger} \hat{a}_{j n}
$$


accounts for bosonic charge, $c$, and spin, $s$, excitations. The energies $\varepsilon_{c, n}=n \varepsilon_{0} / g$ are for $n>0$ depending on the Coulomb interaction parameter $g \simeq 0.2$, while the others scale only with the level spacing: $\varepsilon_{s, \pm|n|}=\varepsilon_{c,-|n|}=|n| \varepsilon_{0}$. None of the bosonic excitations influences the particle number in the single bands and hence one can classify the states of the CNT by a vector $|\vec{N}, \vec{m}\rangle$, where $\vec{N}=\left(N_{+\uparrow}, N_{+\downarrow}, N_{-\uparrow}, N_{-\downarrow}\right)$ determines the fermionic configuration and $\vec{m}$ determines the bosonic excitations (see, e.g., Ref. 20).

The ground states $|\vec{N}, \vec{m}=0\rangle:=|\vec{N}\rangle$ of shell $n$ have $4 n$, $4 n+1,4 n+2$, and $4 n+3$ electrons and can be characterized in terms of the excess spins in band $\{\tilde{r}, \sigma\}$ with respect to the case of equally filled bands: $|n, n, n, n\rangle:=|\cdot, \cdot\rangle, \mid n+$ $1, n, n, n\rangle:=|\uparrow, \cdot\rangle$, or $|n+1, n+1, n, n\rangle:=|\uparrow \downarrow, \cdot\rangle$, etc. (see Table I). For medium-to-large tube radius and far from the charge neutrality point, spin-orbit coupling and exchange effects are not relevant. They can become of interest for large curvatures and will be investigated elsewhere. $\hat{H}_{\mathrm{ext}}^{P / A}$ accounts for external, gate-independent sources of level splitting and holds, at the least, the contribution arising from the external field necessary to control the direction of the contact polarizations.

The leads are described by $\hat{H}_{l}=\sum_{\sigma q} \epsilon_{l q} \hat{c}_{l \sigma q}^{\dagger} \hat{c}_{l \sigma q}$, with $\hat{c}_{l \sigma q}$ annihilating an electron of energy $\epsilon_{l q}$ and of spin $\sigma$ in lead $l$. The density of states in lead $l, D_{l \sigma}(\omega)$, is assumed to be constant over an energy range set by a spin-dependent bandwidth: ${ }^{12,13}$

$$
D_{l \sigma}(\omega)=D_{l \sigma} \theta\left(\omega+W+\sigma \Delta_{\mathrm{St}} / 2\right) \theta\left(W-\sigma \Delta_{\mathrm{St}} / 2-\omega\right) .
$$

It determines the leads polarizations $P_{l}$ at the Fermi level according to $P_{l}=\left(D_{l \uparrow}-D_{l \downarrow}\right) /\left(D_{l \uparrow}+D_{l \downarrow}\right)$.

On the other hand, in order to account for a Stoner splitting $\Delta_{\mathrm{St}}$, the range of available energies in the leads is given by $-\left(W+\sigma \Delta_{\mathrm{St}} / 2\right) \leqslant \omega \leqslant W-\sigma \Delta_{\mathrm{St}} / 2$, where $W$ is the bandwidth at zero Stoner splitting.

Finally the perturbative contribution is $\hat{H}_{T}=$ $\sum_{l \sigma} \int d^{3} r\left(T_{l}(\vec{r}) \hat{\psi}_{\sigma}^{\dagger}(\vec{r}) \hat{\phi}_{l \sigma}(\vec{r})+\right.$ H.c. $)$, allowing for tunneling between CNT and leads. Here $T_{l}(\vec{r})$ is the tunnel coupling, $\hat{\psi}_{\sigma}(\vec{r})$ is the CNT bulk electron operator as given in Ref. 19, and $\hat{\phi}_{l \sigma}(\vec{r})=\int d \epsilon D_{l \sigma}(\epsilon) \sum_{\left.q\right|_{\epsilon}} \phi_{l q}(\vec{r}) \hat{c}_{l \sigma q}$ is the lead electron operator with $\phi_{l q}(\vec{r})$ denoting the corresponding single-particle wave function.

\section{TRANSPORT THEORY}

The current, as any other observable of the transport setup, can be calculated by a trace over the associated operator multiplied by the reduced density matrix $\hat{\rho}(t)=\operatorname{Tr}_{l}\left\{\hat{\rho}_{\text {tot }}(t)\right\}$. Being obtained from the density matrix $\hat{\rho}_{\text {tot }}$ of the total setup upon tracing out the lead degrees of freedom, $\hat{\rho}(t)$ stores the full information about the state of the dot in the presence of the tunnel coupling to the leads. The time evolution of $\hat{\rho}(t)$ follows the Liouville equation, and it can for the stationary state $(\dot{\hat{\rho}}(t)=0)$ be cast into the form (see, e.g., Ref. 21)

$$
0=-i \sum_{a a^{\prime}} \delta_{a b} \delta_{a^{\prime} b^{\prime}}\left(E_{a}-E_{a^{\prime}}\right) \rho_{a a^{\prime}}+\sum_{a a^{\prime}} K_{b b^{\prime}}^{a a^{\prime}} \rho_{a a^{\prime}},
$$
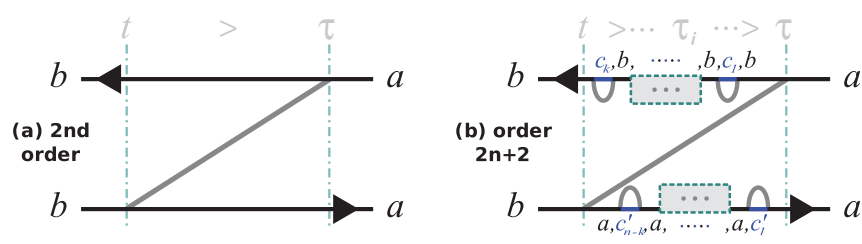

FIG. 2. (Color online) Structure of (a) a sequential tunneling diagram and (b) a level renormalization diagram for a sequential tunneling event. Each "bubble" describes a charge fluctuation. We consider $k$ charge fluctuations (to states $c_{j}$ ) in the final state $b$ and $n-k$ charge fluctuations (to states $c_{i}^{\prime}$ ) in the initial state $a$. We have indicated the time order, as well as the intermediate states on the contours.

taking matrix elements with respect to the many-body eigenstates of the CNT: $\rho_{a a^{\prime}}:=\left\langle a|\hat{\rho}| a^{\prime}\right\rangle$ and $E_{a}:=\langle a|\hat{H}| a\rangle$. Furthermore $K_{b b^{\prime}}^{a a^{\prime}}:=\left\langle b\left|\left[K|a\rangle\left\langle a^{\prime}\right|\right]\right| b^{\prime}\right\rangle$, with the kernel superoperator $K$ arising from the perturbation, i.e., the tunnel coupling between quantum dot and leads. Depending on up to which order $2 n$ in the tunnel coupling $K$ is calculated, one takes into account effects from $2 n$ correlated tunnel events. The most involved part of a perturbative transport calculation is the determination of the kernel $K$.

A fully diagrammatic representation of $K$ has first been proposed in Ref. 15. For example, one contribution to the second-order kernel $\left(K^{(2)}\right)_{b b}^{a a}$ is shown in Fig. 2(a). It relates to the tunnel process $|a\rangle \rightarrow|b\rangle$, represented by the solid line connecting upper and lower parts of the diagram contour. In the following we identify specific terms in all orders of the perturbation series as charge fluctuation processes, which sum up to a Taylor series yielding the intrinsic level renormalization $E_{b a} \rightarrow E_{b a}+h_{\text {int }}^{b a}$ to the energy difference $E_{b a}=E_{b}-E_{a}$, where $h_{\text {int }}^{b a}$ is given in Eq. (7) below.

\section{A. Diagrammatic evaluation of the intrinsic level renormalization}

Recently, it has been recognized ${ }^{21}$ that certain fourth-order diagrams can be related to charge fluctuations in the initial or final state during a tunnel event.

Here we identify a specific class of these charge fluctuation diagrams which, summed up in all orders, yield a whole Taylor series and therewith an actual level shift. Figure 2(b) shows a diagram of order $2 n+2$, contributing to $\left(K^{(2 n+2)}\right)_{b b}^{a a}$, which dresses Fig. 2(a) by $k$ charge fluctuations in the final state $b$ ( $k$ "bubbles" on the upper part of the contour) and $n-k$ charge fluctuations in the initial state $a(n-k$ "bubbles" on the lower part of the contour). We thereby look at fluctuations isolated, in the sense of separated in time, from each other: each bubble must start and end at consecutive times $\tau_{i}$ and $\tau_{i+1}(1 \leqslant i, j \leqslant 2 n-1)$. The electron transfer event is initialized at the earliest time $\tau=\tau_{0}$ and ends at the latest time $\tau_{2 n+1}=t$. This gives $\left(\begin{array}{l}n \\ k\end{array}\right)$ possibilities for the time ordering of the bubbles among the upper and lower parts of the contour. Summing all those plus their hermitian conjugates, the total contribution is, as obtained by applying diagrammatic rules, ${ }^{15}$ 


$$
\begin{aligned}
\longrightarrow & \text { H.c. } \sim \lim _{\eta \rightarrow 0}\left(\begin{array}{l}
n \\
k
\end{array}\right) \operatorname{Re} \frac{2 i}{\hbar} \int d \omega D_{l \sigma}(\omega) f_{l}^{p}(\omega)\left(\frac{1}{p \omega-E_{b a}+i \eta}\right)^{n+1}\left|T_{l \sigma}^{p}(b, a)\right|^{2} \\
& \times\left[\prod_{j=1}^{k} \int d \omega_{j} \frac{D_{l_{j} \sigma_{j}}\left(\omega_{j}\right) f_{l_{j}}^{p_{j}}\left(\omega_{j}\right)\left|T_{l_{j} \sigma_{j}}^{p_{j}}\left(c_{j}, b\right)\right|^{2}}{p_{j} \omega_{j}-E_{c_{j} a}+p \omega+i \eta} \prod_{i=1}^{n-k} \int d \omega_{i} \frac{D_{l_{i} \sigma_{i}}\left(\omega_{i}\right) f_{l_{i}}^{p_{i}}\left(\omega_{i}\right)\left|T_{l_{i} \sigma_{i}}^{p_{i}}\left(c_{i}^{\prime}, a\right)\right|^{2}}{-p_{i} \omega_{i}-E_{b c_{i}^{\prime}}+p \omega+i \eta}\right] .
\end{aligned}
$$

Here $c_{j}$ and $c_{i}^{\prime}$ serve as placeholders for the possible intermediate states; $f_{l}^{p}(\omega):=f^{p}\left(\beta \omega+\beta e V_{l}\right), p= \pm$, where we defined $f^{+}(\omega)=f(\omega)=1 /\left(1+e^{\omega}\right)$, denoting the Fermi function, while $f^{-}(\omega)=f(-\omega)$. The lead indices $l, l_{i}, l_{j} \in$ $\{s, d\}$ are summed over, and the lead energies $\omega, \omega_{i}$, and $\omega_{j}$ are integrated over. The values of $p$ and $\sigma$ are set by fixing the initial and final states $a$ and $b$. Likewise the values of the spin indices $\sigma_{j}$ and $\sigma_{i}$ and of the Fermi function labels $p_{j}$ and $p_{i}$ depend on the intermediate states $c_{j}$ and $c_{i}^{\prime}$ that can be reached from $b$ and $a$, respectively, and are summed over. Finally, there are tunnel matrix elements characterizing transitions on the dot, $T_{l \sigma}^{-(+)}(b, a) \sim T_{l}\left(\vec{r}_{l}\right)\left\langle b\left|\psi_{\sigma}^{(\dagger)}\left(\vec{r}_{l}\right)\right| a\right\rangle\left(\vec{r}_{l}\right.$ characterizes the position of lead $l$ ). A multitude of terms emerges from the expression Eq. (6) when applying the decomposition

$$
\begin{aligned}
& \lim _{\eta \rightarrow 0} \int d \tilde{\omega} \frac{D_{\tilde{l} \tilde{\sigma}}(\tilde{\omega}) f^{\tilde{p}}(\tilde{\omega})}{\tilde{\omega}-\tilde{\mu}+i \eta} \\
& \quad=\int^{\prime} d \tilde{\omega} \frac{D_{\tilde{l} \tilde{\sigma}}(\tilde{\omega}) f^{\tilde{p}}(\tilde{\omega})}{\tilde{\omega}-\tilde{\mu}}-i \pi D_{\tilde{l} \tilde{\sigma}}(\tilde{\mu}) f^{\tilde{p}}(\tilde{\mu})
\end{aligned}
$$

for all fractions and expanding the product ( $f^{\prime}$ denotes a principal part integration; $\tilde{\omega} \in\left\{\omega, \omega_{i}, \omega_{j}\right\}$, etc.). We want to focus on a certain contribution, namely the combination where the fraction containing merely $\omega$,

$$
\left(\frac{1}{p \omega-E_{b a}+i \eta}\right)^{n+1} \equiv \frac{1}{n !} \frac{\mathrm{d}}{d^{n} E_{b a}}\left(\frac{1}{p \omega-E_{b a}+i \eta}\right)
$$

has been replaced by the $\delta$ function and all others by their principal parts.

In terms of $f^{(n)}(\omega)=\frac{d}{d^{n} \omega} f(\omega)$ it reads

$$
\begin{aligned}
& \frac{2 \pi}{\hbar} \frac{1}{n !} f^{(n)}\left(\beta E_{b a}+\beta p e V_{l}\right) D_{l \sigma}\left|T_{l \sigma}^{p}(b, a)\right|^{2} \beta^{n}\left(\begin{array}{l}
n \\
k
\end{array}\right) \\
& \quad \times \operatorname{Re}\left[\prod_{j=1}^{k} \int^{\prime} d \omega_{j} \frac{f^{+}\left(\omega_{j}\right) D_{l_{j} \sigma_{j}}\left|T_{l_{j} \sigma_{j}}^{p_{j}}\left(c_{j}, b\right)\right|^{2}}{\omega_{j}-\beta E_{c_{j} b}-p_{j} \beta e V_{l_{j}}}\right. \\
& \left.\quad \times \prod_{i=1}^{n-k} \int^{\prime} d \omega_{i} \frac{f^{-}\left(\omega_{i}\right) D_{l_{i} \sigma_{i}}\left|T_{l_{i} \sigma_{i}}^{p_{i}}\left(c_{i}^{\prime}, a\right)\right|^{2}}{\omega_{i}-\beta E_{a c_{i}^{\prime}}+p_{i} \beta e V_{l_{i}}}\right] .
\end{aligned}
$$

Exhausting all possibilities of choosing the states $c_{j}$ and $c_{i}^{\prime}$ as well as setting $k=1, \ldots, n$, it is clear that one generates all kinds of terms appearing in an expansion of the power $n$ of a quantity $h_{\text {int }}^{b a}$ defined as

$$
\begin{aligned}
h_{\mathrm{int}}^{b a}= & \sum_{l}\left(\sum_{c}\left|T_{l \sigma}^{p}(c, b)\right|^{2} \int^{\prime} d \omega \frac{f^{+}(\omega) D_{l \sigma}(\omega)}{\omega-\beta E_{c b}-p \beta e V_{l}}\right. \\
& \left.+\sum_{c^{\prime}}\left|T_{l \sigma}^{p}\left(c^{\prime}, a\right)\right|^{2} \int^{\prime} d \omega \frac{f^{-}(\omega) D_{l \sigma}(\omega)}{\omega-\beta E_{a c^{\prime}}+p \beta e V_{l}}\right),
\end{aligned}
$$

where $c$ and $c^{\prime}$ run over all states connected to $|b\rangle$, respectively, $|a\rangle$ via a charge fluctuation. In total, we obtain the $n$th term $\frac{2 \pi}{\hbar} \frac{1}{n !} f^{(n)}\left[\beta\left(E_{b a}+p e V_{l}\right)\right]\left|T_{l \sigma}^{p}(b, a)\right|^{2} D_{l \sigma}\left(\beta h_{\text {int }}^{b a}\right)^{n}$ in the Taylor expansion of a Fermi function $\frac{2 \pi}{\hbar} f\left[\beta\left(E_{b a}+e V_{l}+\right.\right.$ $\left.\left.h_{\text {int }}^{b a}\right)\right]\left|T_{l \sigma}^{p}(b, a)\right|^{2} D_{l \sigma}$. So, effectively, the considered contribution of the initial and final state charge fluctuations renormalizes any energy difference $E_{b a}$ to $E_{b a}+h_{\text {int }}^{b a}$. From comparison with Eq. (6), we can extract a renormalization to a many-body energy $E_{b}$ by looking at all the possible fluctuations on the upper contour:

$$
\delta E_{b}=\sum_{l} \sum_{c}\left|T_{l \sigma}^{p}(c, b)\right|^{2} \int^{\prime} d \omega \frac{f_{l}^{p}(\omega) D_{l \sigma}(\omega)}{p \omega-E_{c b}},
$$

where the values of $p$ and $\sigma$ are settled ${ }^{22}$ once the state $c$ is assigned. For the case of the single impurity Anderson model this expression reduces to the result for the level shift obtained in perturbation theory. ${ }^{10,11}$ Hence Eq. (8) provides a diagrammatic interpretation of these results. As temperature goes to zero, these diagrams diverge logarithmically at the charge-degeneracy points, which gives a parametric selection of these specific diagrams.

For a multilevel quantum dot, the summation over the intermediate virtual states $c$ implies that the amplitude of the level shifts is expected to vary from a resonance peak to the other, as also shown in Figs. 4, 5, and 6. Notice also that for the CNT used in our calculations, the summation over the virtual states also includes states with bosonic excitations as described by the bosonic Hamiltonian $\hat{H}_{B}$ in Eq. (3).

\section{B. Flat band with Stoner shift}

In order to better analyze the contribution of majority and minority spins, we consider for the evaluation of Eq. (7) the case of a flat band including a Stoner splitting $\Delta_{\mathrm{St}}$, i.e, $D_{l \sigma}(\omega)$ as given in Eq. (4). Notice that in order to retain holomorphic functions, one can make use of the Fermi function representation of the step functions, $\theta( \pm x)=\lim _{\beta \rightarrow \infty} f^{\mp}(\beta x)$. Using 
then the residue calculus, ${ }^{23}$ one obtains

$$
\begin{aligned}
h_{\text {int }}^{b a}= & \sum_{l}\left[\sum_{c} D_{l \sigma}\left|T_{l \sigma}^{p}(c, b)\right|^{2} \tilde{\Psi}_{\sigma,-}^{(0)}\left(\beta E_{c b}+p \beta e V_{l}\right)\right. \\
& \left.-\sum_{c^{\prime}} D_{l \sigma}\left|T_{l \sigma}^{p}\left(c^{\prime}, a\right)\right|^{2} \tilde{\Psi}_{\sigma,+}^{(0)}\left(\beta E_{a c^{\prime}}-p \beta e V_{l}\right)\right],
\end{aligned}
$$

where the upper/lower boundary $\pm W-\sigma \Delta_{\mathrm{St}} / 2$ of the integration enters via

$$
\begin{aligned}
\tilde{\Psi}_{\sigma, \pm}^{(0)}(x) \equiv & \operatorname{Re}\left[\Psi^{(0)}\left(0.5+\frac{i x}{2 \pi}\right)\right. \\
& \left.-\Psi^{(0)}\left(0.5+i \frac{x-\beta\left( \pm W-\sigma \Delta_{\mathrm{St}} / 2\right)}{2 \pi}\right)\right]
\end{aligned}
$$

where $\Psi^{(0)}$ is the digamma function. An approximation of this result is obtained elegantly when using Eq. (4) to split the integration range of the integrals in Eq. (7) as

$$
\begin{aligned}
& \int_{-W-\Delta_{\mathrm{St}} / 2}^{W-\Delta_{\mathrm{St}} / 2} d \omega=\int_{-W-\Delta_{\mathrm{St}} / 2}^{-W+\Delta_{\mathrm{St}} / 2} d \omega+\int_{-W+\Delta_{\mathrm{St}} / 2}^{W-\Delta_{\mathrm{St}} / 2} d \omega, \\
& \int_{-W+\Delta_{\mathrm{St}} / 2}^{W+\Delta_{\mathrm{St}} / 2} d \omega=\int_{-W+\Delta_{\mathrm{St}} / 2}^{W-\Delta_{\mathrm{St}} / 2} d \omega+\int_{W-\Delta_{\mathrm{St}} / 2}^{W+\Delta_{\mathrm{St}} / 2} d \omega,
\end{aligned}
$$

and noting that in the region at the top/bottom of the band is $f(\omega)=0 / 1$. Here we get the result

$$
\begin{aligned}
h_{\mathrm{int}}^{b a}= & \sum_{l}\left[\sum_{c} D_{l \sigma}\left|T_{l \sigma}^{p}(c, b)\right|^{2} \tilde{\Psi}_{\downarrow,+}^{(0)}\left(\beta E_{c b}+p \beta e V_{l}\right)\right. \\
& \left.-\sum_{c^{\prime}} D_{l \sigma}\left|T_{l \sigma}^{p}\left(c^{\prime}, a\right)\right|^{2} \tilde{\Psi}_{\uparrow,-}^{(0)}\left(\beta E_{a c^{\prime}}-p \beta e V_{l}\right)\right] \\
& +\sum_{l, c} D_{l \uparrow}\left|T_{l \uparrow}^{p}(c, b)\right|^{2} \ln \left(\frac{W_{\uparrow}+E_{c b}+p e V_{l}}{W_{\downarrow}+E_{c b}+p e V_{l}}\right) \\
& +\sum_{l, c^{\prime}} D_{l \downarrow}\left|T_{l \downarrow}^{p}\left(c^{\prime}, a\right)\right|^{2} \ln \left(\frac{W_{\downarrow}-E_{a c^{\prime}}+p e V_{l}}{W_{\uparrow}-E_{a c^{\prime}}+p e V_{l}}\right),
\end{aligned}
$$

where $W_{\sigma}=W-\sigma \Delta_{\mathrm{St}} / 2$ is the finite bandwidth for the integration over the lead energies. The result is an approximation of Eq. (9), as can be seen when applying $\ln (A / B) \approx$ $\operatorname{Re} \Psi(0.5+i A)-\operatorname{Re} \Psi(0.5+i B)$ in Eq. (10).

The first two terms depend strongly on gate voltage, interaction, and polarization. They correspond to the contribution to the level shift first proposed in Ref. 11.

In contrast, obviously, the last two terms in Eq. (10) vanish if $\Delta_{\mathrm{St}}=0$. Moreover, for $E_{c b}, E_{a c^{\prime}} \ll W_{\sigma}$ they are very weakly dependent on the gate voltage and polarization, but do depend on the saturation magnetization via the Stoner splitting. Following Ref. 5, with tunneling coupling $\Gamma \simeq 1 \mathrm{meV},{ }^{24}$ this contribution can yield for $\mathrm{PdNi}$ contacts the gate-independent part of the level shift of the order of a few hundreds of $\mu \mathrm{eV}$. This is of the same order as the constant shift used to fit the Kondo data in Refs. 3 and 5 but also the TMR data ${ }^{1}$ in Ref. 6. A full account of the interface phase shifts might be necessary for large transparencies and goes beyond the scope of our tunneling Hamiltonian.
However, in the regime where the tunneling model is valid (Coulomb blockade and Kondo regime), tunneling-induced shifts should suffice to explain the experiments.

All in all, we can recast the expression for $h_{\text {int }}^{b a}$ in the form

$$
h_{\mathrm{int}}^{b a}=h_{0}^{b a}+\Delta h^{b a}\left(\Delta_{\mathrm{St}}\right) .
$$

The first term depends logarithmically on the gate voltage and the interaction strength, while $\Delta h^{b a}$ is the contribution due to $\Delta_{\mathrm{St}}$, which depends on the finite bandwidth $W_{\sigma}$ of $D_{l \sigma}(\omega)$ and will be almost gate and interaction independent and thus contribute in the same way as the terms $h_{\mathrm{ext}}^{P / A}$ induced by $\hat{H}_{\mathrm{ext}}^{P / A}$.

Notice that if the states $a, b, c$, and $c^{\prime}$ have electron-hole symmetry partners $a_{\star}, b_{\star}, c_{\star}$, and $c_{\star}^{\prime}$, there exists the mirror transition $b_{\star} \rightarrow a_{\star}$, with $a_{\star}$ now being the final state and $b_{\star}$ the initial state. Thus the contributions from the gate-dependent part of the charge fluctuations to $c_{\star}$ are negative, and those to $c_{\star}^{\prime}$ are positive, such that $h_{0}^{b_{\star} a_{\star}}=-h_{0}^{b a}$. This preserves the mirror symmetry of all curves when $\Delta_{\mathrm{St}}=0$ [cf. Fig. 3(a)].

\section{LINEAR CONDUCTANCE AND TMR RESULTS}

We consider the linear bias regime for spin polarized transport across a CNT without band offset (cf. Table I).

Figure 3 shows the conductances for parallel (pink solid) and antiparallel (blue dashed) lead magnetizations under the influence of the distinct types of level shifts, along with the corresponding TMR curves. Throughout this work we assume equal lead polarizations $P_{s}=P_{d}=P$ and the coupling asymmetry $\gamma=\Gamma_{s} / \Gamma_{d} \sim\left|T_{s}\right|^{2} /\left|T_{d}\right|^{2}$, with the actual values of $\gamma$ given at each plot.

In the absence of any Zeeman shift, Fig. 3(a) shows the typical conductance peak patterns expected for the degeneracies according to Table I, for both a second-order (sequential tunneling) and a fourth-order (cotunneling, pair tunneling, single charge fluctuations, etc. $)^{21}$ truncation in the calculation of the kernel $K$. Second-order theory predicts a constant TMR value,${ }^{14}$ but this changes upon inclusion of higher-order effects. Within fourth-order perturbation theory, the TMR exhibits an oscillatory gate voltage dependence, albeit the variation is small. ${ }^{25}$ Notice that the increase at the edges of the plot is nonphysical, stemming from a limitation of the numerical calculation to six $(4 n-1 \leqslant N \leqslant 4 n+4)$ charge states.

To visualize the TMR mechanism from Figs. 1(c) and 1(d), we include in Fig. 3(b) merely a constant shift from an external source, $\left|h_{\text {ext }}^{P}\right|=\left|h_{\text {ext }}^{A}\right|>k_{B} T$ (this can arise from the external field which controls the direction of contact polarization in TMR measurements, when for the measurement of $G_{P}$ the field is swept back to the point where $G_{A}$ has been measured before).

As explained above, among the formerly degenerate lowestlying states, the ones with maximum spin projection $S_{z}$ are selected as ground states, as highlighted in Table I. At the first two peaks transport is thus mediated by $\uparrow$ electrons, and at the last two peaks by $\downarrow$ electrons. This fact breaks the symmetry in the conductance curve for a parallel configuration which favors transport via the $\uparrow$ electron channel. For an antiparallel magnetization of the leads, both spin species are minority charge carriers in one of the two leads and thus provide 
(a) no level shifts, $Y=1$

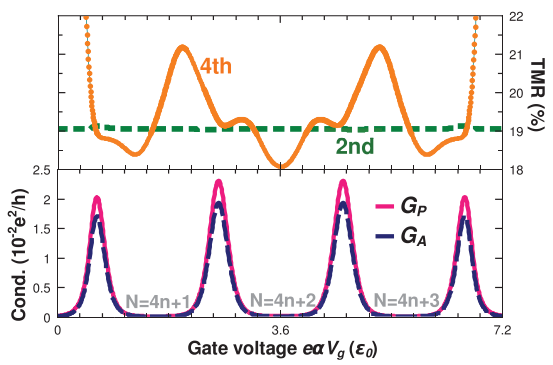

(d) intrinsic level shifts, $\gamma=1, \Delta_{S t}=0$

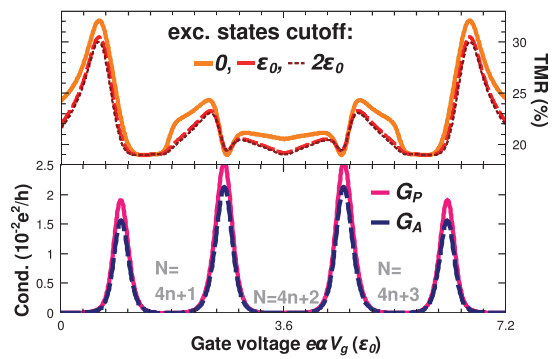

(b) constant external shift $/ h^{P}{ }_{\text {ext }} /=\mid h^{A}{ }_{\text {ext }} /, \gamma=1$

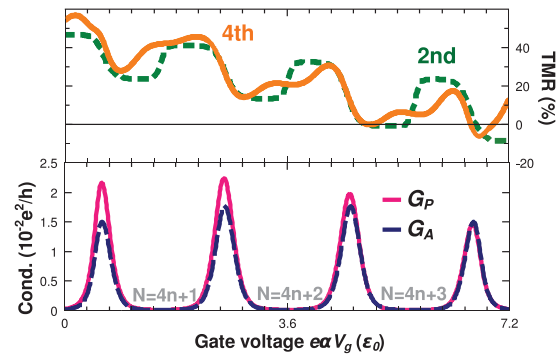

(e) intrinsic level shifts, $\gamma=1, \Delta_{S t}=0.2 W$

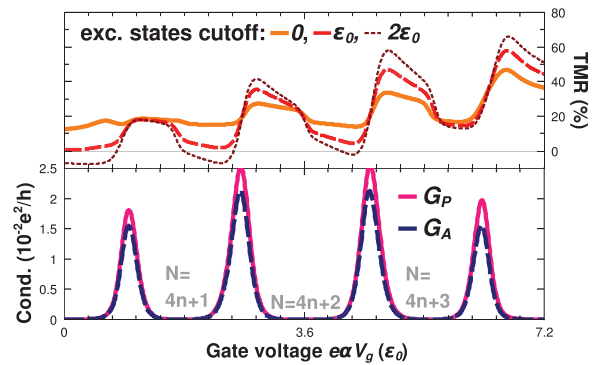

(c) constant external shift $/ h^{P}$ ext $|>| h^{A}{ }_{\text {ext }} /, \gamma=1$

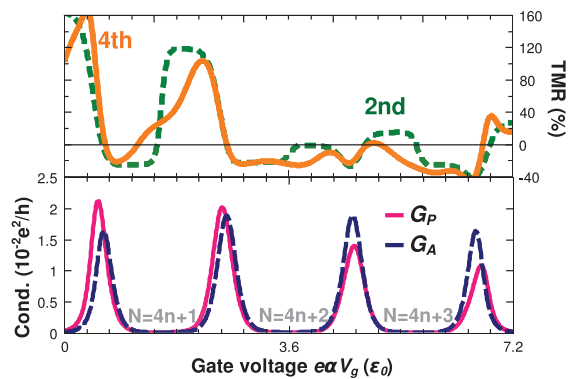

(f) intrinsic shifts plus $/ h^{P}{ }_{\text {ext }} /=\mid h^{A}$ ext $/$

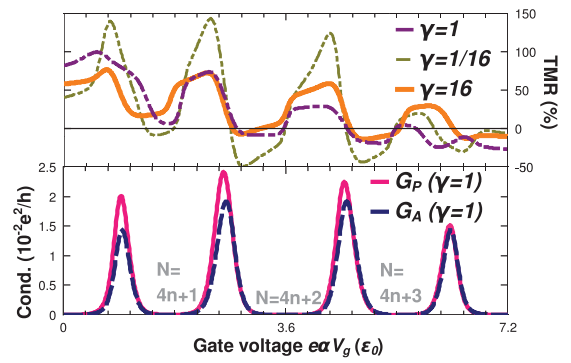

FIG. 3. (Color online) Parallel $\left(G_{P}\right)$ and antiparallel $\left(G_{A}\right)$ conductance along with the resulting tunneling magneto-resistance (TMR) for a CNT of $500 \mathrm{~nm}$ in length $\left(\varepsilon_{0}=3.35 \mathrm{meV}\right)$ and charging energy $E_{c}=6.7 \mathrm{meV}$. The thermal energy was set to $k_{B} T=0.3 \mathrm{meV}$, the lead polarization to $P=0.4$, the tunnel broadening to $\hbar \Gamma_{s}=3 \mu \mathrm{eV}$, and the bandwidth to $W=3 \mathrm{eV}$. (a) For a full fourth-order calculation a mirror-symmetric TMR slightly oscillating around a value of $20 \%$ is obtained. (b) An equal splitting $h_{\mathrm{ext}}^{P}=h_{\mathrm{ext}}^{A}=0.4 \mathrm{meV}$ causes gate asymmetry and negative values of the TMR by the mechanism shown in Fig. 1(c). (c) For $h_{\mathrm{ext}}^{P}=2 h_{\mathrm{ext}}^{A}=0.8$ meV, the regularity of the curve is broken and the negative TMR mechanism shown in Fig. 1(b) comes into play. (d) TMR and conductance for $\Delta_{\mathrm{St}}=0$. We find electron-hole symmetry perfectly preserved, also upon inclusion of virtual excited states (with cutoff set to $\varepsilon_{0}$ and $2 \varepsilon_{0}$ ). (e) Same as panel (d) but for $\Delta_{\mathrm{St}}=0.2 \mathrm{~W}$, which breaks electron-hole symmetry. Including the influence of the virtual excited states negative TMR can be reached. (f) Combining intrinsic shifts with external splitting. An asymmetric coupling to the leads can enlarge $(\gamma<1)$ or diminish $(\gamma>1)$ the TMR effect.

equally weak transport channels, such that the symmetry in the conductance curve is preserved. In Fig. 3(c) we still neglect intrinsic shifts, but assume $\left|h_{\text {ext }}^{P}\right|>\left|h_{\text {ext }}^{A}\right|\left(>k_{B} T\right.$, e.g., due to stray fields) to bring also the first negative TMR mechanism, Fig. 1(b), into play. It overlays the effects observed in Fig. 3(b). The shift in the peak positions of parallel and antiparallel conductance peaks enforces a change of the TMR from positive to negative value from the first peak on.
Because there is no significant change in the curves of Figs. 3(b) and 3(c) upon inclusion of the fourth-order effects and the numerical implementation of the gate-dependent contribution $h_{0}^{b a}$ implies a summation over a large number of states for a CNT quantum dot, in the remaining figures we only show second-order curves with all level shifts fully included.

In Figs. 3(d) and 3(e) we include the finite level shifts which arise from our transport theory and investigate the impact of (a) intrinsic level shifts, cutoff 0

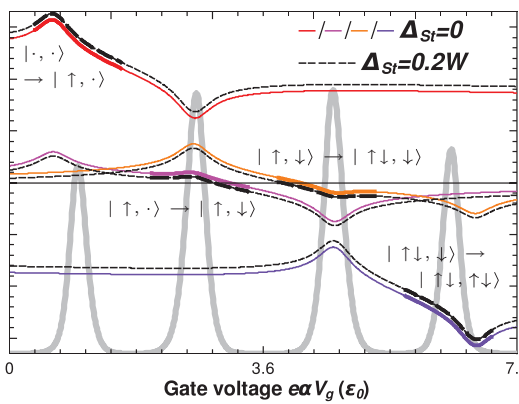

(b) intrinsic level shifts, cutoff $\varepsilon_{0}$

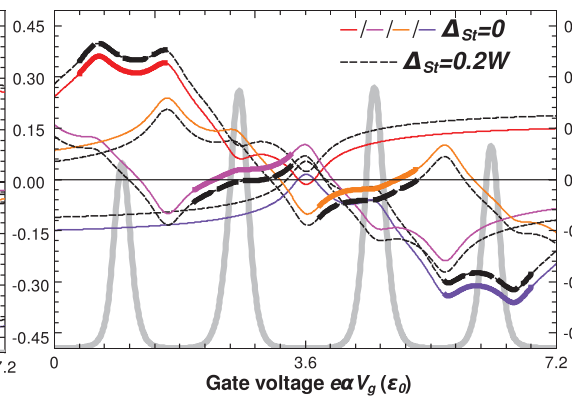

(c) intrinsic level shifts, cutoff $2 \varepsilon_{0}$

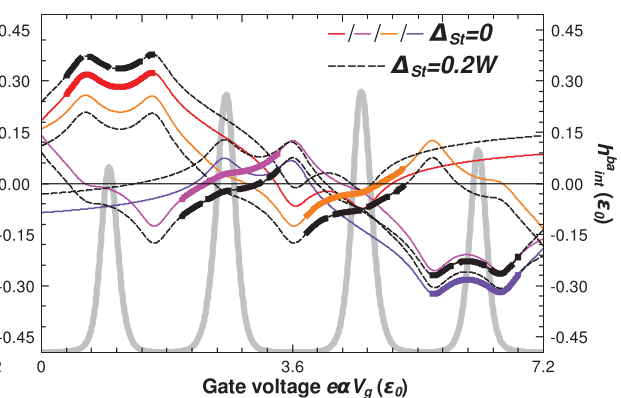

FIG. 4. (Color online) Gate-voltage dependence of the intrinsic level shift $h_{\text {int }}^{\text {ba }}$, Eq. (7), for exemplary electron-hole symmetric transitions, see state labels in panel (a). The electron-hole symmetry is nicely reflected in the curves for $\Delta_{\mathrm{St}}=0$. Moreover, it becomes obvious that $\Delta_{\mathrm{St}}$ acts opposite to an external Zeeman splitting, i.e., increases (decreases) the energy difference for $\uparrow(\downarrow)$ transitions. Raising the energy cutoff for the inclusion of excited states, more and more transitions to virtual states will be contributing to the charge fluctuations for the exemplary transitions. (a) Only fluctuations to ground states. (b) Fluctuations to all states within the range $\varepsilon_{0}$. (c) All states within the range $2 \varepsilon_{0}$. 
(a) difference of energy shifts, spin 0 states

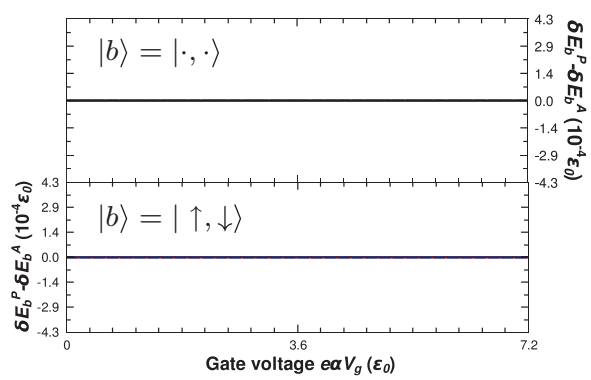

(b) difference of energy shifts, spin $1 / 2$ states

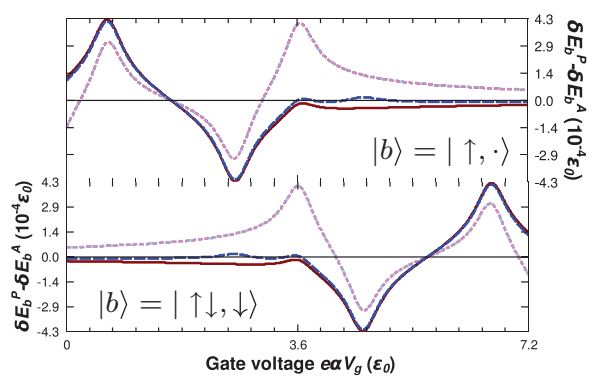

(c) difference of energy shifts, spin 1 states

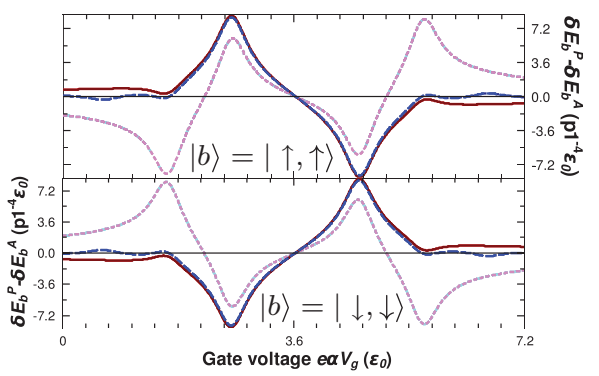

FIG. 5. (Color online) Gate-voltage dependence of the difference of energy shifts $\delta E_{b}^{P}-\delta E_{b}^{A}$ [see Eq. (8)] for states with spin 0 (a), spin $\pm 1 / 2$ (b), and spin \pm 1 (c). Notice the pronounced influence of virtual states including bosonic excitations.

excited states for $\Delta_{\mathrm{St}}=0$ and $\Delta_{\mathrm{St}}=0.2 \mathrm{~W}$, respectively. In the latter case we find that inclusion of virtual transitions to excited states can even yield negative TMR. Notice that, due to the presence of both fermionic and bosonic excitations, the number of available states increases rapidly with energy. For excitations with energy up to $2 \varepsilon_{0}$ one has already 716 possible states. In Fig. 3(d), we find the TMR already nicely converged at the cutoff of $2 \varepsilon_{0}$, while in Fig. 3(e) the trend to convergence can be identified. Note that going beyond a cutoff of $2 \varepsilon_{0}$ is numerically very demanding. Interestingly, the intrinsic gatedependent contribution, Fig. 3(d), distinguishes itself from all former contributions by preserving the mirror symmetry in the conductances and the TMR curve for $\Delta_{\mathrm{St}}=0$. The reason is that a given electron-hole symmetry in the tunneling results in an electron-hole symmetry for any charge fluctuation (see Sec. IV). This is no longer true if $\Delta_{\text {St }} \neq 0$, Fig. 3(e), where we observe a behavior similar to that of a constant external shift but of opposite $\operatorname{sign},{ }^{5}$ i.e., $\Delta_{\text {St }} \neq 0$ induces a gate constant positive (negative) shift, for $\uparrow(\downarrow)$-mediated transitions, which also breaks the electron-hole symmetry. These statements

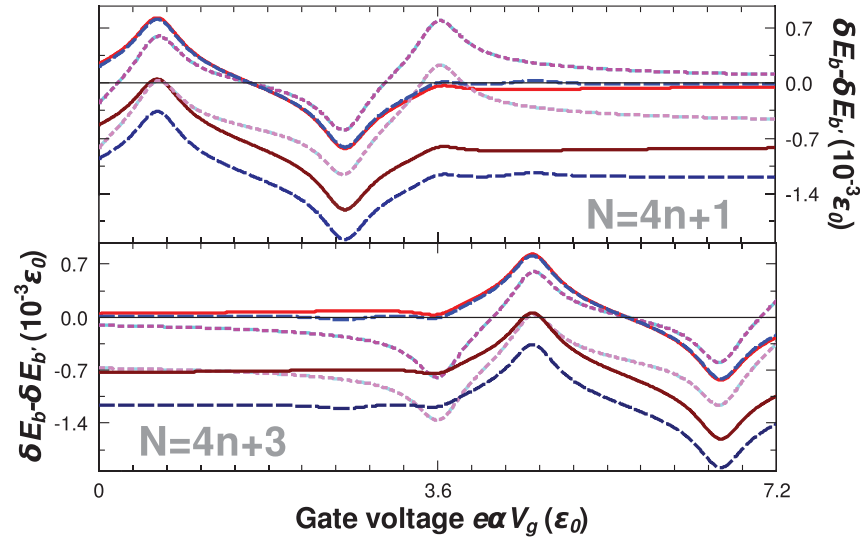

FIG. 6. (Color online) Gate-voltage dependence of the difference of energy shifts $\delta E_{b}-\delta E_{b^{\prime}}$ for parallel configuration for the two states $b=|\uparrow, \cdot\rangle$ and $b^{\prime}=|\downarrow, \cdot\rangle(N=4 n+1$, upper panel $)$ and $b=$ $|\uparrow \downarrow, \uparrow\rangle$ and $b^{\prime}=|\uparrow \downarrow, \downarrow\rangle(N=4 n+3$, lower panel). Notice the pronounced influence of virtual states including bosonic excitations. For the legend, see Fig. 5.

are confirmed by Figs. 4(a)-4(c) which show the shifts $h_{\text {int }}^{b a}$ (Eq. (7), the quantity determining the shift of position of the conductance peaks) for selected electron-hole symmetric transitions $|b\rangle \rightarrow|a\rangle$. Due to the orbital degeneracy of the $\mathrm{CNT}$, the shifts are seen to differ from peak to peak. The trend to convergence is obvious, in particular, around the resonances the respective transitions belong to (curves thickened in the respective gate voltage region).

Finally, Fig. 3(f) combines the impact of the intrinsic shifts with an equal extrinsic splitting $\left|h_{\text {ext }}^{P}\right|=\left|h_{\text {ext }}^{A}\right|$. The intrinsic effects suffice to change the TMR curve, Fig. 3(b), to a shape observed in experiments, ${ }^{1}$ though due to our limitation to the weak coupling regime, quantitative agreement cannot be expected. An asymmetric coupling is found to affect the curve quantitatively, while the qualitative shape is retained. Thereby, $\gamma<1(\gamma>1)$ relates to an increased (decreased) coupling to the drain contact - the one in which the parallel and antiparallel configurations differ from each other [see Fig. 1(a)]—and enhances (suppresses) the TMR effect. Even for the very small values of $\Gamma_{s}$ used here to justify lowest-order perturbation theory, the difference between Figs. 3(b) and 3(f) reveals a marked influence of the intrinsic, tunneling induced, level shifts.

The dramatic influence of the excited states, and among these, in particular, the bosonic excitations, is further demonstrated in Figs. 5 and 6.

In Fig. 5 the difference of energy shifts $\delta E_{b}^{P}-\delta E_{b}^{A}$ [see Eq. (8)] is plotted versus gate voltage for the same states as those used in Fig. 4. Notice that this quantity vanishes for states with total spin 0 . For nonzero spin, the shifts exhibit a characteristic gate dependence, which proves to be most sensitive to the presence of bosonic excitations: omitting them (Fig. 5, magenta dotted + cyan dash-dotted) influences the curves not only quantitatively but also qualitatively. Compared to that effect, it plays only a minor role whether the cutoff is chosen to be $\varepsilon_{0}$ (blue solid) or $2 \varepsilon_{0}$ (red dashed), and the curves for $\Delta_{\mathrm{St}}=0$ and $\Delta_{\mathrm{St}} \neq 0$ practically fall upon one another. In perfect similarity to an externally induced splitting, there is a 
factor of 2 in magnitude between the differences of the energy shifts for the spin- $\frac{1}{2}$ and the spin- 1 states.

In Fig. 6 the quantity $\delta E_{b}-\delta E_{b^{\prime}}$ is shown for the case of parallel configuration, for the two states $b=|\uparrow, \cdot\rangle$ and $b^{\prime}=|\downarrow, \cdot\rangle$ (upper plot) and $b=|\uparrow \downarrow, \uparrow\rangle$ and $b^{\prime}=|\uparrow \downarrow, \downarrow\rangle$ (lower plot). These shifts for states with 1 and 3 excess electrons are those which have been measured, e.g., in the Kondo experiments of Refs. 3 and 5. For $\Delta_{\mathrm{St}}=0$ the result is already converged, as the curves for cutoff energies $\varepsilon_{0}$ and $2 \varepsilon_{0}$ are mostly identical. For $\Delta_{\mathrm{St}}=0.2 \mathrm{~W}$ the energy difference acquires a negative offset.

\section{CONCLUSIONS}

We analyzed the impact of different kinds of effective Zeeman shifts in magnetically coupled multilevel quantum dots, obtaining a characteristic gate dependence and the possibility of negative TMR. In particular, we have provided a systematic way of including the important effects of tunneling-induced level shifts in a transport calculation by identifying a subclass of diagrams, to all orders in the tunneling Hamiltonian, describing charge fluctuations. This infinite class of diagrams is seen to correspond to a perturbative renormalization of the dot spectrum, an effect which is indeed expected on physical grounds. In conventional Feynman diagrammatics, these terms arise from summing up the second-order dot electron self-energy in the Dyson equation. Importantly, this concept generalizes to higher orders: virtual charge fluctuations during inelastic cotunneling (appearing first in sixth order) give a renormalization of the inelastic cotunneling threshold, as already experimentally observed in Ref. 26. In general, a TMR signal will be influenced by many parameters relevant to the given device. Nevertheless, following the lines of the analysis given here for a CNT, it should be possible to disentangle the importance of the various contributions.

\section{ACKNOWLEDGMENTS}

We acknowledge fruitful discussions with C. Strunk, S. Pfaller, and J. Hauptmann as well as the support of the DFG under Program SFB 689.
${ }^{1}$ S. Sahoo, T. Kontos, J. Furer, C. Hoffmann, M. Gräber, A. Cottet, and C. Schönenberger, Nat. Phys. 1, 99 (2005).

${ }^{2}$ A. N. Pasupathy, R. C. Bialczak, J. Martinek, J. E. Grose, L. A. K. Donev, P. L. McEuen, and D. C. Ralph, Science 306, 86 (2004).

${ }^{3}$ J. R. Hauptmann, J. Paaske, and P. E. Lindelof, Nat. Phys. 4, 373 (2008).

${ }^{4}$ L. Hofstetter, A. Geresdi, M. Aagesen, J. Nygård, C. Schönenberger, and S. Csonka, Phys. Rev. Lett. 104, 246804 (2010).

${ }^{5}$ M. Gaass, A. K. Hüttel, K. Kang, I. Weymann, J. von Delft, and C. Strunk, Phys Rev. Lett. 107, 176808 (2011).

${ }^{6}$ A. Cottet and M.-S. Choi, Phys. Rev. B 74, 235316 (2006).

${ }^{7}$ A. Cottet, T. Kontos, S. Sahoo, H. T. Man, M.-S. Choi, W. Belzig, C. Bruder, A. F. Morpurgo, and C. Schönenberger, Semicond. Sci. Technol. 21, 78 (2006).

${ }^{8}$ A. Brataas, Y. V. Nazarov, and G. E. W. Bauer, Phys. Rev. Lett. 84, 2481 (2000).

${ }^{9}$ W. Wetzels, G. E. W. Bauer, and M. Grifoni, Phys. Rev. B 72, 020407(R) (2005).

${ }^{10}$ F. D. M. Haldane, Phys. Rev. Lett. 40, 416 (1978).

${ }^{11}$ J. Martinek, Y. Utsumi, H. Imamura, J. Barnás, S. Maekawa, J. König, and G. Schön, Phys. Rev. Lett. 91, 127203 (2003).

${ }^{12}$ J. Martinek, M. Sindel, L. Borda, J. Barnás, R. Bulla, J. König, G. Schön, S. Maekawa, and J. von Delft, Phys. Rev. B 72, 121302(R) (2005).
${ }^{13}$ M. Sindel, L. Borda, J. Martinek, R. Bulla, J. König, G. Schön, S. Maekawa, and J. von Delft, Phys. Rev. B 76, 045321 (2007).

${ }^{14}$ S. Koller, L. Mayrhofer, and M. Grifoni, New J. Phys. 9, 348 (2007).

${ }^{15}$ H. Schoeller and G. Schön, Phys. Rev. B 50, 18436 (1994).

${ }^{16}$ M. Braun, J. König, and J. Martinek, Phys. Rev. B 70, 195345 (2004).

${ }^{17}$ R. P. Hornberger, S. Koller, G. Begemann, A. Donarini, and M. Grifoni, Phys. Rev. B 77, 245313 (2008).

${ }^{18}$ P. Trocha, I. Weymann, and J. Barnás, Phys. Rev. B 80, 165333 (2009).

${ }^{19}$ L. Mayrhofer and M. Grifoni, Phys. Rev. B 74, 121403(R) (2006).

${ }^{20}$ L. Mayrhofer and M. Grifoni, EPJ B 56, 107 (2007).

${ }^{21}$ S. Koller, M. Grifoni, M. Leijnse, and M. R. Wegewijs, Phys. Rev. B 82, 235307 (2010).

${ }^{22} T_{l \sigma}^{p}(c, b)$ is zero unless the electronic configurations of $b$ and $c$ differ by one electron of spin $\sigma$. Then, $p=\operatorname{sgn}\left(N_{c}-N_{b}\right)$, with $N_{a}:=\langle a|\hat{N}| a\rangle$.

${ }^{23}$ Equivalently to $\int^{\prime} d \omega f^{p}(\omega) D_{l \sigma}(\omega) /(\omega-\mu)$ one can consider $\operatorname{Im} \int_{0}^{\infty} d t \int_{-\infty}^{\infty} d \omega D_{l \sigma}(\omega) f_{l}^{p}(\beta \omega) e^{i(\omega-\mu) t}$.

${ }^{24}$ Notice that $\Gamma_{l} \sim D_{l} T_{l}^{2}$ and $\Gamma=\sum_{l} \Gamma_{l}$.

${ }^{25}$ I. Weymann, J. König, J. Martinek, J. Barnas, and G. Schön, Phys. Rev. B 72, 115334 (2005).

${ }^{26}$ J. V. Holm, H. I. Jørgensen, K. Grove-Rasmussen, J. Paaske, K. Flensberg, and P. E. Lindelof, Phys. Rev. B 77, 161406(R) (2008). 